\title{
100GHz Electrically Tunable Planar Bragg Grating via Nematic Liquid Crystal Overlay towards Reconfigurable WDM Networks
}

\author{
Faisal Rafiq Mahamd Adikan, James C. Gates, Huw E. Major, Corin B.E. Gawith, Peter G.R. Smith \\ Optoelectronics Research Centre (ORC), University of Southampton, SO17 1BJ Southampton, United \\ Kingdom \\ Phone: $+44(0) 2380594530$ \\ Fax: $+44(0) 2380593149$ \\ E mail:mfra@orc.soton.ac.uk \\ Andriy Dyadyusha, Malgosia Kaczmarek \\ School of Physics and Astronomy, University of Southampton, SO17 1BJ Southampton, United \\ Kingdom \\ lan J.G. Sparrow \\ Stratophase Ltd, Unit A7, The Premier Centre, Premier Way, Romsey, Hampshire SO51 9DG
}

\begin{abstract}
Novel liquid crystal-based integrated optical devices with $>140 \mathrm{GHz}$ electrical tuning are presented for application towards reconfigurable wavelength division multiplexing (WDM) networks. Initial results with Bragg wavelength tuning covering five $25 \mathrm{GHz}$ WDM channel spacing have been achieved with $170 \mathrm{~V}$ (peak-to-peak) sinusoidal voltages applied across electro-pattemed ITO-covered glass electrodes

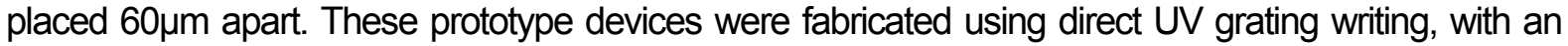
evanescent field coupling into a liquid crystal overlay through an etched window. Electrically controlled liquid crystal birefringence modifies the waveguide effective index, resulting in Bragg wavelength shift. Merck 18523 nematic liquid crystals are used, exhibiting compatible refractive index values to that of silica $\left(n_{0}=1.44, n_{e}=1.49\right.$ at $\left.\lambda=1550 \mathrm{~nm}\right)$. Homeotropic alignment of the liquid crystal is provided by application of a surfactant layer.

The inherent refractive index sensitivity of our etched direct-UV-written structures allows observation of previously unreported liquid crystal surface-behaviour, such as multi-threshold points during variation of the applied field. Continued optimisation based on evanescent field penetration, electrode layout, and surface interaction will allow implementation towards a variety of novel liquid crystal applications and devices. For example, a cascaded architecture of these integrated liquid crystal devices operating at different Bragg wavelengths would pave the way towards true colorless add/drop modules for dense optical networks.
\end{abstract}

Keywords: Liquid crystal, planar Bragg gratings, direct UV writing, tunable optical devices, reconfigurable optical networks.

Principal author biography: Rafiq Adikan is currently pursuing a doctorate at the Optoelectronics Research Centre (Southampton). He studied L-band erbium-doped fibre amplifiers for his Masters degree at the University of Malaya (Malaysia) and holds a Bachelor degree in electrical and electronics engineering from the University of Manchester Institute of Science and Technology (UMIST). 